\title{
Planificación territorial y mitigación de impacto de tsunami en Chile después del 27 Febrero $2010^{1}$
}

The role of Urban Planning in Mitigating Tsunami in Chile after February 27th, 2010

\author{
Marie Geraldine Herrmann Lunecke
}

\section{Filiación}

Universidad Diego Portales, Chile

E mail: geraldine.herrmann@udp.cl

Primera versión recibida en: 31 de marzo, 2016

Última versión recibida en: 14 de junio, 2016

\section{Resumen}

Esta investigación analiza el rol de la planificación territorial en la mitigación de impacto de tsunami en Chile, específicamente en respuesta al tsunami del año 2010. Se analizan las normativas y recomendaciones de mitigación de impacto de tsunami desarrolladas en Chile a escala nacional y las medidas propuestas a escala local en los Planes de Reconstrucción del Borde Costero (PRES) a partir de la revisión de documentos de planificación y la realización de entrevistas semi-estructuradas con actores claves.

Este artículo revela que la planificación territorial en Chile no ha considerado suficientemente la amenaza de tsunami, pese a que Chile es un país muy expuesto a maremotos. Los resultados de esta investigación muestran que la elaboración de medidas de mitigación de impacto de tsunami en los PRES se ha realizado en un marco regulatorio nacional débil y que las medidas de mitigación propuestas en los distintos PRES son de carácter no vinculante, enfocándose principalmente en soluciones ingenieriles "anti-tsunámicas". Otros instrumentos de planificación como son la zonificación de riesgo o relocalización se han propuesto solamente en unos pocos PRES, sin instrumentos adecuados para su implementación y sin una participación ciudadana activa.

\section{Palabras clave}

Planificación urbana; mitigación impacto tsunami; reconstrucción; resiliencia

\begin{abstract}
This article analyses the role of urban planning in mitigating the impact of tsunami in Chile, especially in response to the tsunami of February 27th, 2010. It examines the policies to reduce the impact of tsunami developed at the national level and analyses and compares tsunami mitigation measures proposed at the local level in recent reconstruction plans (PRES) for coastal towns and villages, through a detailed policy review and semi structured interviews with key actors.

This article shows that tsunami mitigation measures proposed in the non-binding PRES are being developed in a weak national regulatory framework and focus strongly on "anti-tsunami" engineering solutions. Other available planning tools like risk zoning and relocation have only been proposed in a few coastal reconstruction plans without suitable instruments for their implementation and lacking an active participation of citizens.
\end{abstract}

\section{Keywords}

Urban planning; tsunami impact mitigation; reconstruction; resilience

1 Esta investigación fue financiada por el Fondo de Investigación Semilla de la Universidad Diego Portales. 


\section{Sumario \\ $1 \quad$ Metodología \\ 2 Resultados \\ 2.1 Respuesta de la planificación territorial en Chile a escala nacional frente a la amenaza de tsunami \\ 2.2 Mitigación de impacto de tsunami en Chile a escala local a través de los PRES \\ 3 Discusión y Conclusiones \\ Referencias}

\section{Introducción}

El sábado 27 Febrero 2010 a las 03:34 horas el centro sur de Chile fue afectado por un terremoto de 8.8 grados Richter, seguido de un tsunami. El terremoto fue el sexto más fuerte registrado en el mundo, afectando un área con más de 8 millones de personas, causando 521 muertes, la desaparición de 56 personas y un daño material de alrededor de 30.000 millones de dólares (OECD, 2013). Fueron devastados el área metropolitana del Gran Concepción, 5 ciudades con más de 100.000 habitantes y más de 900 pueblos y comunidades rurales y costeras (MINVU, 2010: p.1). Alrededor de 80.000 hogares fueron destruidos y 110.000 hogares sufrieron daños severos.

El terremoto fue seguido por un tsunami que causó graves daños a lo largo de 500 kilómetros de costa desde Navidad hasta Tirúa, y en la isla Juan Fernández a 600 kilómetros de la costa chilena. Un rasgo distintivo del tsunami chileno fue su extrema variabilidad en altura, destructividad y tiempo de llegada de olas (EERI, 2010: p.5). En general, la primera ola del tsunami llegó a menos de 30 minutos del terremoto, y en la mayoría de las áreas se observaron tres a cuatro oleajes. La tercera y cuarta ola fueron las más grandes, llegando entre 90 minutos y 4 horas después del sismo. Los niveles más altos de agua se registraron entre 10 a 12 metros sobre el nivel del mar (EERI, 2010: p.5). En relación a los tsunamis, las edificaciones más afectadas fueron las construcciones en madera y las construcciones de mampostería sin, o con poco reforzamiento, sufriendo severos daños por las fuertes cargas hidrodinámicas y el impacto de escombros flotantes. Las edificaciones de hormigón, en cambio, registraron un buen comportamiento estructural durante el tsunami, incluso cuando los niveles de inundación alcanzaban por encima del segundo piso (EERI, 2010: p.6).

Dada la magnitud del impacto de este tipo de desastre socio-natural, ¿cómo podemos aumentar nuestra resiliencia costera frente a futuros tsunami?

Este trabajo plantea que un instrumento clave para mejorar la resiliencia comunitaria frente a desastres socio-naturales debe ser la planificación urbana. En este sentido cabe destacar que en Chile la planificación territorial ha tenido un rol fundamental en la mitigación de impacto de terremotos durante los últimos 80 años. Chile tiene un terremoto sobre 8.0 grados Richter en promedio cada 17 años. Sin embargo, gracias al desarrollo de regulaciones sismo resistentes, las construcciones en Chile están hoy satisfactoriamente preparadas para que al menos sus estructuras resistan los terremotos ${ }^{2}$. Así, en el terremoto del 27 de Febrero 2010 (27F) los

\footnotetext{
${ }^{2}$ Chile cuenta hoy con una clasificación de zonas sísmicas y tipos de suelo y con normas de cálculo antisísmico que regulan las estructuras de los edificios. Así, la Ordenanza General de Urbanismo y Construcciones incluye regulaciones básicas respecto al diseño sismo resistente de los edificios e indica tipo de suelos donde la construcción está restringida. Otro instrumento importante en Chile es la norma NCh 433 que contempla el cálculo antisísmico de edificios, aprobada como Norma Provisional en 1966 y como Norma Oficial en 1972.
} 
mayores daños se registraron en construcciones de adobe y mampostería no reforzada, construidas antes de la introducción de regulaciones antisísmicas en Chile.

Se observa claramente en Chile que fuertes sismos en áreas pobladas han dado paso al desarrollo de regulaciones sismo-resistentes (Fig. 1). De hecho, la primera Ley y Ordenanza General de Construcciones y Urbanización en Chile fue creada como respuesta al terremoto de Talca de 1928 (Sarrazín, 1985: p.25; Ibáñez, 1985: p.27). Así, el terremoto de Talca inicia en Chile no sólo los estudios en ingeniería antisísmica, conocimiento tomado en gran parte de la tecnología extranjera, sino también la planificación urbana en Chile.

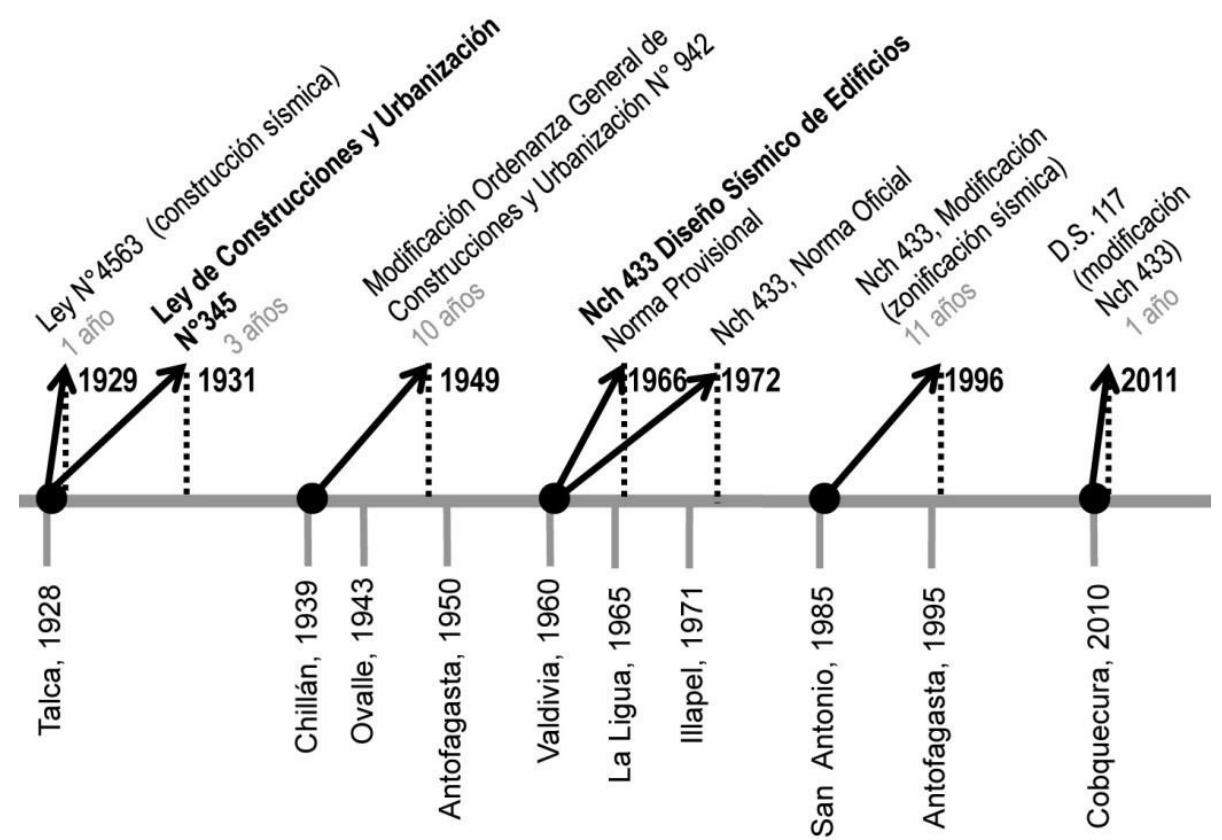

Fig. 1 Respuesta de la planificación territorial en Chile a escala nacional ante terremotos Fuente: Elaboración propia en base a Sismología Universidad de Chile, Sarrazín (1985) e Ibáñez (1985)

Tal como ha ocurrido exitosamente en el caso de los terremotos, la planificación urbana debería ser también un instrumento clave para mitigar los efectos devastadores de un tsunami.

¿Cuál ha sido en Chile la respuesta de la planificación territorial frente a la amenaza de tsunami a escala nacional y a escala local?

La presente investigación tiene como objetivo analizar el rol de la planificación territorial en la mitigación de impacto de tsunami en Chile, específicamente en respuesta al tsunami del año 2010. Con este fin, se revisan las recientes normativas y recomendaciones de mitigación de impacto de tsunami desarrolladas en Chile a escala nacional y se analizan y comparan en detalle las medidas de mitigación de impacto de tsunami propuestas en los 24 Planes de Reconstrucción del Borde Costero (PRES) de la Región del Maule y Región del Bío Bío, a partir de la revisión de documentos de planificación y la realización de entrevistas semi-estructuradas con actores quienes participaron en la elaboración de los PRES.

Los resultados de esta investigación muestran que la elaboración de medidas de mitigación de impacto de tsunami en los recientes planes locales de reconstrucción se ha realizado en un marco regulatorio nacional débil. Hasta hoy, la única normativa relevante desarrollada en Chile tras la catástrofe del 27F a escala nacional es la modificación de la Ordenanza General de 
Urbanismo y Construcciones (OGUC) en el año 2011, que incluye por primera vez las zonas de inundación por tsunami como áreas de riesgo (D.S. N9, modificación OGUC). Sin embargo, la OGUC no establece parámetros para la ocupación y el manejo de zonas de riesgo por tsunami: no establece normas o estándares para guiar a los municipios en la planificación, ni restringe o prohíbe usos en dichas zonas. Debido a la falta de regulación a escala nacional, las medidas de mitigación de impacto de tsunami difieren altamente entre los distintos PRES. Mientras solo unas pocas comunas restringen o prohíben equipamiento clave y uso residencial en zonas de inundación por tsunami, la mayoría de los PRES únicamente propone estrategias de evacuación o infraestructura de mitigación.

Otro problema a escala local es que las medidas de mitigación de efectos de tsunami propuestas en los distintos PRES son en general de carácter no vinculante, enfocándose principalmente en soluciones ingenieriles "anti-tsunámicas". Sin embargo, instrumentos de planificación como son la zonificación de riesgo - restringiendo, por ejemplo infraestructura clave en zonas de inundación por tsunami (colegios, hospitales, etc.)- o la relocalización de vivienda se han propuesto solamente en unos pocos PRES, sin instrumentos adecuados para su implementación y sin una participación ciudadana activa.

\section{Metodología}

La presente investigación analiza el rol de la planificación territorial en la mitigación de impacto de tsunami en Chile, específicamente en respuesta al terremoto y tsunami del año 2010. Con este fin, esta investigación emplea diferentes herramientas metodológicas de investigación cualitativa y cuantitativa. Primero, se revisan las recientes normativas y recomendaciones de mitigación de impacto de tsunami desarrolladas en Chile a escala nacional tras el tsunami del 2010 (Ley General de Urbanismo y Construcciones, Ordenanza General de Urbanismo y Construcciones, Ley de Concesiones Marítimas, Ley de Puertos, Ley de Concesiones Públicas, Proyectos de Ley ingresados al Congreso entre 2010-2015). Posteriormente, se analizan y comparan en detalle las políticas y medidas de mitigación de impacto de tsunami propuestas en los 24 Planes de Reconstrucción del Borde Costero (PRES) de la Región del Maule y Región del Bío Bío, a partir de la realización de entrevistas semi-estructuradas y cuestionarios en terreno a actores quienes participaron en la elaboración de los PRES.

Entre junio 2011 y abril 2012 se aplicaron un total de 50 entrevistas semi-estructuradas y cuestionarios a informantes claves del sector público, privado y civil. Los cuestionarios recogieron información detallada respecto a: situación general antes de la catástrofe del $27 \mathrm{~F}$ (instrumentos de planificación territorial, medidas de mitigación de tsunami); catástrofe del $27 \mathrm{~F}$ (alerta y evacuación de tsunami); planes de reconstrucción del borde costero (contenido general, actores, participación ciudadana, tiempo y costo de elaboración, financiamiento, etc.); medidas de mitigación de impacto de tsunami propuestas en los distintos planes de reconstrucción: planes de inundación por tsunami, estrategias de evacuación incluyendo rutas de evacuación, infraestructura "anti-tsunami", normativa urbanística tsunami resistente, áreas de riesgo por tsunami, restricciones de uso de suelo en zonas de inundación por tsunami, relocalización desde zonas de riesgo hacia zonas seguras, zonas de seguridad por tsunami, evacuación vertical por tsunami, y, seguros obligatorios en zonas de inundación por tsunami; actores y participación ciudadana en la elaboración de medidas de mitigación de impacto de tsunami; y, conformidad de las medidas de mitigación de efectos de tsunami con normas a escala nacional.

El procesamiento y análisis de la información fue realizado mediante un análisis de contenido simple, así como un análisis de frecuencias de cada categoría de respuesta, a través del paquete de análisis de datos Excel. 


\section{Resultados}

\subsection{Respuesta de la planificación territorial en Chile a escala nacional frente a la amenaza de tsunami}

La planificación territorial en Chile no ha considerado suficientemente la amenaza de tsunami, pese a que Chile es un país muy expuesto a maremotos. En los últimos 440 años se han registrado en Chile 11 tsunamis destructivos, lo que significa que Chile tiene un tsunami destructivo en promedio cada 40 años (Tabla 1). Cabe destacar que en Chile el largo del borde marítimo es de casi 4.200 kilómetros, sin embargo, la longitud total de costa del territorio continental más las islas suma 83.850 kilómetros (Giménez, Pantoja \& Troncoso, 2010: p.6).

Tabla 1 Tsunamis destructivos en Chile

\begin{tabular}{|c|c|c|c|c|}
\hline Año & Magnitud & Epicentro & Muertos & $\begin{array}{l}\text { Tipo de } \\
\text { Tsunami }\end{array}$ \\
\hline 1570 & 8.3 & Concepción & 2000 & TD \\
\hline 1575 & 8.5 & Valdivia & 200 & TD \\
\hline 1604 & 8.5 & Arica & 120 & TD \\
\hline 1615 & 8.8 & Arica & - & TM \\
\hline 1647 & 8.5 & Santiago & 600 & - \\
\hline 1657 & 8.8 & Concepción & 34 & TD \\
\hline 1730 & 8.7 & Valparaíso & 3000 & TD \\
\hline 1751 & 8.5 & Concepción & 65 & TM \\
\hline 1819 & 8.3 & Copiapó & 133 & TD \\
\hline 1822 & 8.5 & Vallenar & 800 & TM \\
\hline 1835 & 8.5 & Concepción & 120 & TD \\
\hline 1837 & 8.0 & Valdivia & 12 & TM \\
\hline 1868 & 8.5 & Tacna, Perú (costa) & 500 & TD \\
\hline 1877 & 8.5 & Iquique & 34 & TD \\
\hline 1906 & 8.2 & Valparaíso & 3000 & TM \\
\hline 1914 & 8.2 & Talca-Curicó & - & - \\
\hline 1918 & 8.2 & Copiapó & 6 & TM \\
\hline 1922 & 8.5 & Vallenar & 800 & TM \\
\hline 1928 & 8.3 & Talca & 279 & $\mathrm{~T}$ \\
\hline 1939 & 8.3 & Talca-Chillán & 5648 & - \\
\hline 1943 & 8.2 & Ovalle & 8 & $T$ \\
\hline 1950 & 8.3 & Antofagasta & - & - \\
\hline 1960 & 9.5 & Valdivia & $\begin{array}{c}1000 / 1655 \\
\text { (TSU) / } \\
\text { (TOT) }\end{array}$ & TD \\
\hline 1965 & 7.4 & La Ligua & 280 & - \\
\hline 1971 & 7.5 & Illapel & 85 & TM \\
\hline 1985 & 8.0 & San Antonio (costa) & 177 & $\mathrm{~T}$ \\
\hline 1995 & 8.0 & Antofagasta (costa) & 3 & $T$ \\
\hline 2010 & 8.8 & Cobquecura (costa) & $\begin{array}{c}156 / 525 \\
\text { (TSU) / } \\
\text { (TOT) }\end{array}$ & TD \\
\hline
\end{tabular}

Fuente: Elaboración propia en base a Sismología Universidad de Chile 
La zona costera se define como una franja de ancho variable situada en la zona de contacto de mar, tierra y atmósfera, condición que le otorga características ambientales de fragilidad y vulnerabilidad (Andrade, Arenas y Guijón, 2008: p.24). Por otro lado, la zona costera ha sido históricamente un espacio muy atractivo para el asentamiento humano a través de segundas residencias, turismo, pesca, transporte, industria, agricultura, etc., resultando en la concurrencia de múltiples usos y frecuentemente en conflictos territoriales y ambientales (Andrade, Arenas y Guijón, 2008: p.24). En general, se observa en Chile una debilidad de la institucionalidad vigente y la normativa que regula las zonas costeras, así como una falta de coordinación entre los múltiples organismos públicos y privados. En Chile el borde costero es regulado por múltiples organismos como las Municipalidades, la Subsecretaría de Marina, el Ministerio de Bienes Nacionales, el Ministerio de Obras Públicas, etc. Así, las zonas costeras son un territorio de difícil delimitación con una gran cantidad de normas de ordenamiento territorial y organismos sectoriales con traslape de atribuciones (Andrade, Arenas y Guijón, 2008: p.28), lo cual dificulta su regulación.

El desastre del 27F mostró claramente que Chile no estaba preparado para un tsunami: no contaba ni con adecuados sistemas de alerta y evacuación de tsunami, ni con instrumentos de planificación territorial para mitigar los impactos de maremoto. Así, el 27F la Oficina Nacional de Emergencia del Ministerio del Interior (ONEMI) canceló erróneamente la alerta de tsunami, ya que el Servicio Hidrográfico y Oceanográfico de la Armada de Chile (SHOA) ${ }^{3}$ no fue capaz de evaluar adecuadamente la amenaza de tsunami por falta de conocimientos técnicos. Asimismo, la caída de los sistemas de comunicación, con excepción de la radio, y la estructura administrativa altamente centralizada de Chile dificultaron una adecuada respuesta de alerta y evacuación frente al tsunami. Las autoridades regionales y las autoridades municipales no contaban ni con el poder, ni con la competencia para manejar la emergencia. De hecho, gran parte de la población realizó la evacuación del borde costero por iniciativa propia inmediatamente después del terremoto, sin haber sido alertada por las autoridades. Es ejemplar el caso de una niña de 12 años en Juan Fernández, quién tocando una campana por iniciativa propia alertó a la población del maremoto.

En general, la respuesta de la planificación territorial en Chile frente a la amenaza de tsunami ha sido muy lenta y reciente. En 1941 comienza la instalación de mareógrafos en la costa chilena y en 1959 el Departamento de Navegación e Hidrografía de la Armada se integra como miembro del Sistema Internacional de Alarma de Tsunamis del Pacífico. Si bien en 1931 la primera Ordenanza General de Construcciones y Urbanización en Chile considera "precauciones contra maremotos" (Art. 178), éstas ya no figuran en la Ordenanza General de Construcciones de 1949. Recién en 1964 se organiza y se pone en funcionamiento el Sistema Nacional de Alerta de Maremotos (SNAM) y en 1974 se crea la ONEMI. Cabe destacar que a partir de 1997 el SHOA comienza a elaborar las Cartas de Inundación por Tsunami (cartas CITSU) para la costa de Chile, que permite definir los niveles máximos de inundación por tsunami esperados para las principales zonas urbanas y portuarias del litoral. Hasta la fecha de hoy se han elaborado en Chile alrededor de 35 cartas CITSU de inundación por tsunami, y faltan aún muchas por elaborar, especialmente para pueblos costeros. Los mapas CITSU muestran que los niveles de inundación por tsunami llegan en Chile desde 6 hasta 19 metros sobre el nivel del mar, siendo la cota de inundación promedio alrededor de 11 a 12 metros. Cabe destacar que el área de inundación del maremoto del $27 \mathrm{~F}$ coincidió en la mayoría de las localidades con la zona de inundación definida previamente por las cartas CITSU, las cuales por lo tanto, predijeron con bastante exactitud las zonas de riesgo por tsunami.

\footnotetext{
${ }^{3}$ En Chile el Servicio Hidrográfico y Oceanográfico de la Armada de Chile (SHOA) está a cargo del Sistema Nacional de Alerta de Maremotos (SNAM) y debe evaluar la posibilidad de un tsunami, estando conectado directamente con el Centro de Alerta de Tsunami del Pacífico (PTWC) en Hawaii.
} 
Uno de los principales objetivos de las cartas CITSU es tener una aplicación directa tanto en la planificación territorial como en la generación de planes de evacuación y protección civil. Sin embargo, en Chile las cartas CITSU no fueron tomadas en consideración en la planificación territorial hasta después del tsunami del 27F. Antes del año 2010 las cartas CITSU fueron sólo consultadas para emplazar señalética de evacuación de tsunami, la que se colocó en algunas ciudades costeras tras el tsunami del Océano índico del año 2004, el cual generó una momentánea conciencia a nivel mundial acerca de la amenaza de maremotos.

Se observa entonces, que antes de la catástrofe del 27 Febrero 2010 las normativas y los instrumentos de planificación territorial en Chile no incluían regulaciones o recomendaciones para mitigar los efectos de tsunamis, pese a que el Servicio Hidrográfico y Oceanográfico de la Armada (SHOA) había estado haciendo desde 1997 Cartas de Inundación por Tsunami. Recién como respuesta al tsunami del 27F, se observa en Chile un impulso incipiente por generar políticas públicas urbanas a escala nacional que reduzcan el daño ocasionado por los maremotos y comienzan a proponerse algunas normativas y recomendaciones para mitigar sus efectos (Fig. 2).

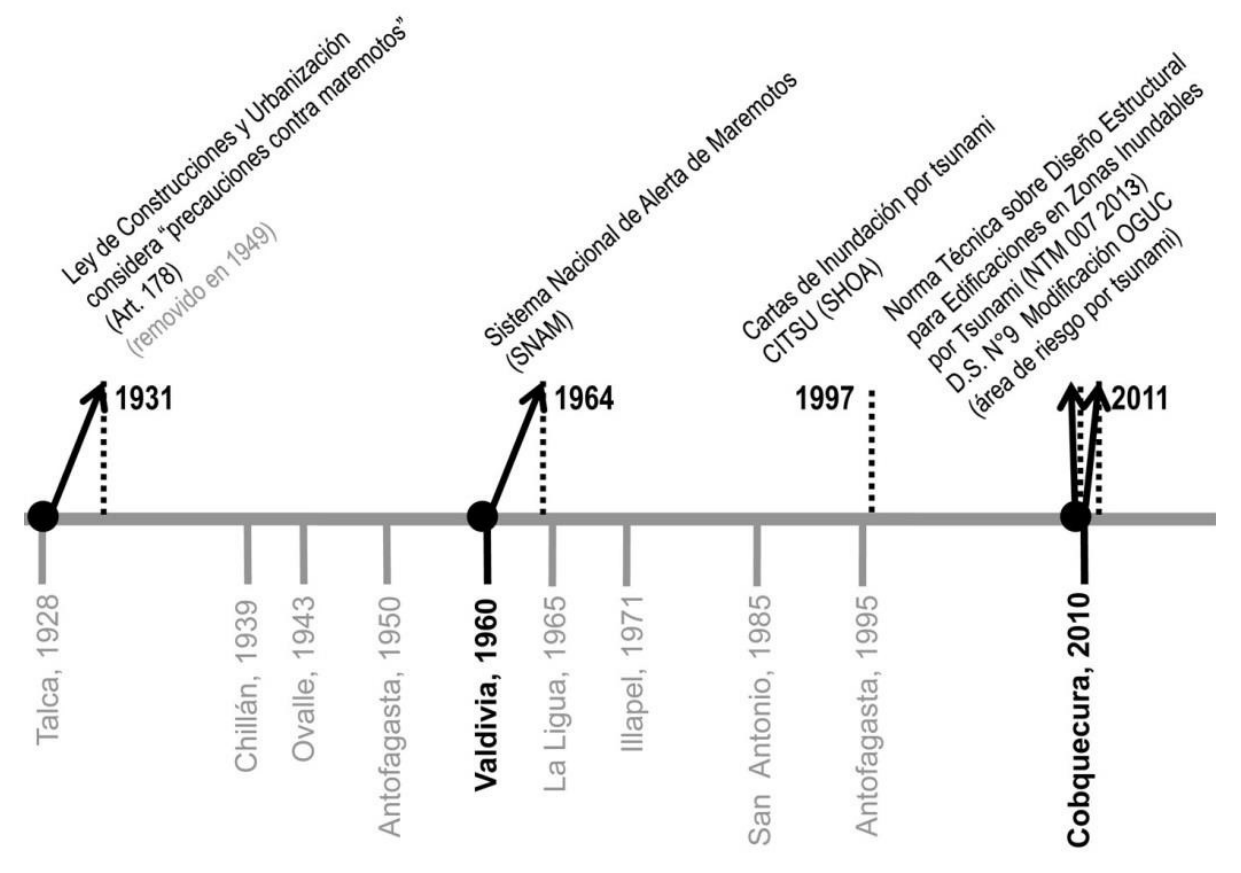

Fig. 2 Respuesta de la planificación territorial en Chile a escala nacional ante tsunamis Fuente: Elaboración propia en base a Sismología U. Chile, SNAM, SHOA (2012) y MINVU (2011)

Así, fue propuesta el año 2010 la denominada Ley de Tsunamis, que modifica la Ley General de Urbanismo y Construcciones, estableciendo que las obras de urbanización y edificación para el borde costero deberán cumplir con los estándares que establezca la Ordenanza General en lo relativo a "las condiciones de resistencia y mitigación de los efectos de maremotos o tsunamis, tales como vías de evacuación en edificios y urbanizaciones, infraestructuras de protección y materialidad de los primeros pisos de las edificaciones" (modificación Ley General de Urbanismo y Construcciones, Art. 105). No obstante, esta propuesta fue congelada.

En esta misma línea, el Ministerio de Vivienda y Urbanismo (MINVU) desarrolla el año 2013 una norma técnica sobre Diseño Estructural para Edificaciones en Zonas Inundables por Tsunami (NTM 007 2013). Esta norma técnica establece que edificaciones no residenciales deben tener 
estructuras tsunami-resistentes, y que edificaciones residenciales deben construirse sobre estructuras de soporte o sobre un relleno protegido, de tal forma que la zona de habitación se ubique sobre la cota de inundación. Cabe señalar que esta norma técnica se refiere al documento estadounidense Guidelines for Design of Structures for Vertical Evacuation from Tsunamis (2008) de FEMA (Federal Emergency Management Agency de EEUU) y considera expresamente como referencia a la Ordenanza de Construcciones Oficial de Honolulu 20022010 (Revised Ordinances Honolulu). No obstante, expertos en Hawaii ${ }^{4}$-como la Directora del International Tsunami Information Center-, señalan que la Ordenanza de Honolulu está obsoleta y que en Estados Unidos se están desarrollando actualmente normas de diseño de edificación tsunami resistente (ASCE 7 Standard), por lo cual la norma técnica chilena en desarrollo estaría tomando como referente un documento obsoleto.

Cabe destacar, que el año 2011 se incluyen por primera vez en la Ordenanza General de Urbanismo y Construcciones las zonas de inundación por tsunami como áreas de riesgo (D.S. $\mathrm{N}^{\circ} 9$, modificación OGUC). En las áreas de riesgo se limita determinado tipo de construcciones por razones de seguridad contra desastres naturales, que "requieran para su utilización la incorporación de obras de ingeniería o de otra índole suficientes para subsanar o mitigar tales efectos" (OGUC, Artículo $\mathrm{N}^{\circ} 2.1 .17$ ). Con el objetivo de permitir a los gobiernos locales incorporar las zonas de riesgo a sus planes reguladores comunales de forma más rápida, el gobierno chileno desarrolló un procedimiento para aprobar modificaciones de los Planes Reguladores Comunales mediante la ley de Sismos y Catástrofes (Ley $\mathrm{N}^{\circ}$ 16.282, Art. 27), reduciendo el procedimiento de 7 años a 2 años e incluso 6 meses (MINVU, 2012). De acuerdo a esta ley de catástrofes las modificaciones de planes reguladores comunales solamente deberán ser aprobadas por los Consejos Comunales, enviadas al MINVU que cuenta para su pronunciamiento con un plazo de 90 días, y firmadas mediante un decreto supremo. En total 31 municipalidades postularon a modificaciones de los Planes Reguladores Comunales mediante la Ley de Sismos y Catástrofes, de las cuales 12 fueron aprobadas. ${ }^{5}$ Cabe señalar que mediante este procedimiento la participación ciudadana y los estudios de impacto ambiental no son requeridos. Asimismo, las modificaciones de los planes reguladores deben contar obligatoriamente con la aprobación de un especialista antisísmico, pero sólo opcionalmente con un estudio de riesgo. Esto resulta cuestionable, dado que se requiere de estudios de riesgo para poder definir zonas de riesgo y es deseable la participación de la ciudadanía en la elaboración de medidas de mitigación de impacto de desastres naturales.

\subsection{Mitigación de impacto de tsunami en Chile a escala local a través de los PRES}

En el contexto de un marco regulatorio nacional débil para mitigar los impactos por tsunami: ¿Cuál ha sido la respuesta de la planificación territorial en Chile a escala local para reducir la vulnerabilidad frente a los maremotos?

En Chile, tras la catástrofe del 27F, los principales instrumentos de planificación urbana de reconstrucción han sido los subsidios habitacionales y los planes de reconstrucción (PRES, PRE y PRU). Los planes de reconstrucción territorial en Chile constituyen planes maestros de carácter no vinculante con una cartera de proyectos, que han sido elaborados por municipalidades, el MINVU, universidades y consultoras urbanas y cofinanciados por empresas privadas. Se observa que en la Octava Región del Bío Bío la elaboración de planes de reconstrucción fue financiada por el sector público, mientras que en la Séptima Región del Maule fue financiada por el sector público en conjunto con el sector privado.

4 Entrevista con Laura Kong, Directora, International Tsunami Information Center Hawaii (ITIC) (6 Junio 2012); Entrevista con Gary Y.K. Chock, President, Martin \& Chock, Inc. (7 J unio 2012).

5 Entrevista con Angela Prado, Departamento de Planificación y Normas Urbanas, División de Desarrollo Urbano, Ministerio de Vivienda y Urbanismo (25 Mayo 2012). 
Los PRES establecen un marco de diseño urbano, definiendo para cada localidad su estructura urbana, uso de suelo, sistema de transporte y espacios públicos, infraestructura y área de crecimiento y expansión urbana. Asimismo, muchos planes de reconstrucción incluyen políticas públicas y proyectos que reducen el impacto de tsunami y otros desastres naturales como remoción en masa e inundación de río. Durante el año 2010 se elaboraron en Chile en total 137 planes de reconstrucción: 25 Planes de Reconstrucción del Borde Costero (PRES), 2 Planes de Reconstrucción Interiores para las ciudades de Talca y Curicó (PRE) y 110 Planes de Regeneración Urbana (PRU) para pequeñas y medianas localidades costeras e interiores (MINVU, 2012). De los 25 Planes de Reconstrucción del Borde Costero (PRES) 1 plan fue elaborada en la Región de Valparaíso (Juan Fernández), 6 planes en la Región del Maule (Duao, lloca, La Pesca, Constitución, Pelluhue y Curanipe) y 18 planos en la Región del Bío Bío (Cobquecura, Caleta Perales, Purema, Dichato, Caleta del Medio, Caleta los Morros, Caleta Tumbes, Lirquén, Penco, Talcahuano, Puerto Sur I sla Santa María, Caleta Lo Rojas, Llico, Tubul, Lebu, Quidico, Tirúa e Isla Mocha).

La presente investigación revisa y analiza en detalle las medidas de mitigación de efectos de tsunami propuestas en los distintos PRES en la Región del Maule y Región del Bío Bío, las dos regiones más severamente afectadas por el tsunami del año 2010. Las medidas de mitigación de impacto de tsunami son clasificadas por este estudio en: planos de inundación por tsunami (cota máxima de inundación por tsunami); estrategias de evacuación incluyendo rutas de evacuación por tsunami; infraestructura "anti-tsunami"; normativa urbanística tsunami resistente; áreas de riesgo por tsunami; restricciones de uso de suelo en zonas de inundación por tsunami; relocalización desde zonas de riesgo hacia zonas seguras; zonas de seguridad por tsunami; evacuación vertical por tsunami; y, seguros obligatorios en zonas de inundación por tsunami (Fig. 3).

Los resultados muestran que todas las localidades en la Región del Maule y la Región del Bío Bío cuentan hoy con planos de inundación por tsunami. Sin embargo, mientras que Talcahuano (2000), Constitución (2002), Lebu (2002), Penco (2000) y Lirquén (2000) tenían cartas CITSU desde los años 2000/2002, las otras localidades confeccionaron cartas de inundación por tsunami recién el año 2010 como respuesta al maremoto del 27F. Es importante señalar que las cartas de inundación por tsunami en Chile están siendo elaboradas no solamente por el SHOA, sino también por las universidades y consultoras privadas sin un método estandarizado, y en algunos casos, sin ser de acceso público y gratuito.

Todos los PRES de la Región del Maule y Región del Bío Bío establecen una cota máxima de inundación por tsunami, que varía entre los 5 metros y los 14 metros sobre el nivel del mar. Sin embargo, como los planos de inundación por tsunami han sido desarrollados por diferentes instituciones sin un método estandarizado, las cotas máximas de inundación varían en un $21 \%$ de los PRES. Por ejemplo, para el caso de Llico la Universidad Católica determina 10 metros como nivel máximo de inundación por tsunami, mientras que la Universidad del Bío Bío establece 6 metros como cota de inundación por tsunami. Las cotas de inundación por tsunami difieren también en el caso de Tubul $(U C=10 \mathrm{~m}, \mathrm{U}$. Bío Bío=6,5m), Talcahuano (UC=10m, U. Bío Bío $=7,5 \mathrm{~m})$, Caleta Tumbes (PRES $=10 \mathrm{~m}, \mathrm{U}$. Bío Bío=7,5m) y Dichato $(\mathrm{UC}=10 \mathrm{~m}$, U. Bío Bío=6,5m).

Una vez definidas las zonas de inundación por tsunami: ¿Cuáles han sido las medidas de mitigación de tsunami propuestas en los distintos planes de reconstrucción? ¿Se han establecido áreas de riesgo por tsunami, restricciones de uso en zonas de riesgo, estrategias de relocalización, infraestructura de mitigación, normativas urbanísticas tsunami resistente y/o estrategias de alerta y evacuación? 


\section{¿Qué medidas de mitigación de impacto de tsunami fueron propuestas en los PRES de la Región del Maule y Region del Bío Bío?}

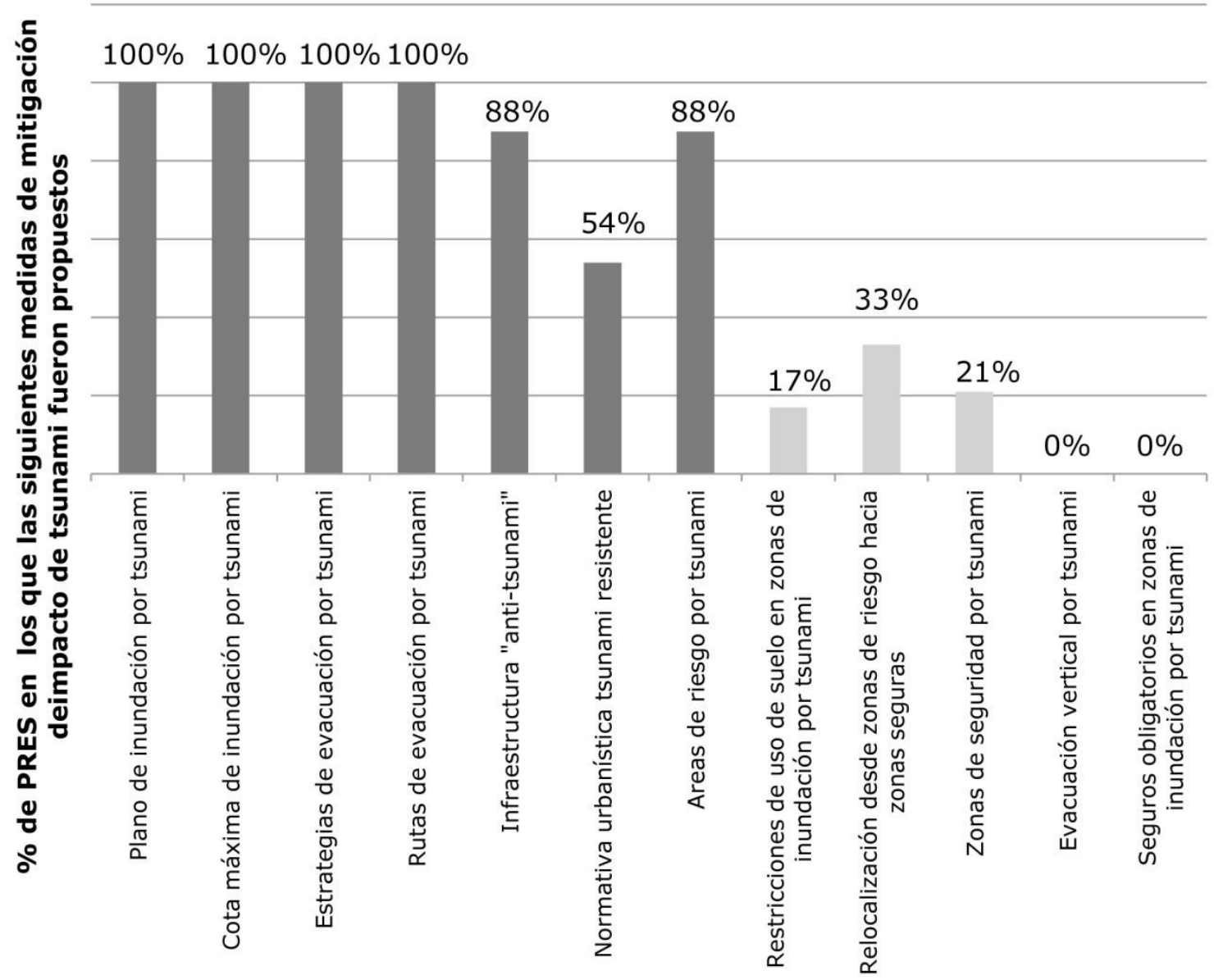

\section{Políticas y medidas de mitigación de impacto de tsunami}

Fig. 3 Respuesta de la planificación urbana en Chile a escala nacional ante tsunamis

Fuente: Elaboración propia basado en PRES y entrevistas realizadas con actores regionales y locales años $2011 / 2012$

Se observa en primer lugar que todos los PRES definen rutas de evacuación a partir de la vialidad existente. Más aún, el PRES de Pelluhue propone vías peatonales hacia zonas seguras y el PRES de Constitución establece que las vías de evacuación deben tener vereda y calle al mismo nivel, soterramiento de redes eléctricas e iluminación fotovoltaica. En todas las localidades deben elaborarse además planes de evacuación y protección civil, que tienen que ser supervisados en cada localidad por la ONEMI a través de los Comités Locales de Emergencia, en conformidad con la Metodología Básica para la Elaboración de un Plan Comunal de Respuesta ante Tsunami (ONEMI, 2001). Cabe destacar, que en Chile la topografía costera facilita la evacuación, ya que en muchos casos las playas cuentan con cerros aledaños o cercanos. Sin embargo, muchas rutas de evacuación cruzan hoy terrenos privados enrejados, que no son públicamente accesibles, lo que podría generar un grave problema durante una emergencia de tsunami. Asimismo, ningún PRES en la Región del Maule y Región del Bío Bío sugiere en caso de tsunami la evacuación vertical hacia la edificación en altura. 
Resalta también que casi todos los PRES (88\%) de la Región del Maule y Región del Bío Bío proponen infraestructura de mitigación "anti-tsunami", específicamente muros de mitigación, parques de mitigación, bosques de mitigación y/o dunas de mitigación. Un ejemplo es el caso de Constitución, donde se proyecta un parque de mitigación de 35 hectáreas con un costo estimado de 20.000 millones de pesos chilenos; otro ejemplo es Dichato con un bosque de mitigación de 2 hectáreas y de un costo estimado de 550 millones de pesos chilenos. Sin embargo, los anchos de ambos parques son insuficientes para reducir efectivamente la energía de un futuro tsunami, dado que un bosque de mitigación debe tener un ancho mínimo de 50 metros (Forbes and Broadhead, 2007: p.10; Tanaka et al., 2007: p.35).

Asimismo, destaca que la mitad (54\%) de los PRES propone normativas urbanísticas tsunami resistente en zonas de inundación por tsunami. Estas normativas establecen, por ejemplo, que las viviendas en zona de riesgo de tsunami deben tener fundaciones con primeros pisos de albañilería o hormigón armado (Talcahunao y Constitución) o ser "vivienda anti-tsunami palafítica" (Región del Bío Bío). Sin embargo, dado que no existen normas técnicas y estándares tsunami-resistentes a escala nacional, las normativas propuestas a escala local varían significativamente entre las distintas localidades y no están fundadas en estudios técnicos.

En conclusión, se muestra que en las localidades costeras de la Región del Maule y del Bío Bío la mitigación de impacto de tsunami post $27 \mathrm{~F}$ se ha centrado en la infraestructura anti-tsunami ( $88 \%$ de los PRES), las estrategias de evacuación (100\% de los PRES) y la normativa urbanística tsunami resistente (54\% de los PRES).

En cambio, otros instrumentos de planificación como son la zonificación de riesgo restringiendo, por ejemplo infraestructura clave en zonas de inundación por tsunami (colegios, hospitales, etc.) - o la relocalización de la vivienda se han propuesto solamente en unos pocos PRES. Si bien todos los planes de reconstrucción - exceptuando Duao/lloca/La Pesca- definen sus áreas de riesgo por tsunami basadas en los recientes planos de inundación por tsunami, la regulación y planificación de estas áreas varía significativamente entre las distintas localidades, lo cual nuevamente se explica por la falta de normas y estándares a escala nacional. La mayoría de las ciudades, incluyendo Talcahuano con más de 160.000 habitantes, sólo usa las áreas de riesgo por tsunami como base para el diseño de estrategias de evacuación por tsunami. De hecho, únicamente 4 de los 24 PRES (17\%) restringen equipamiento clave y uso residencial en las zonas de inundación por tsunami (Constitución, Pelluhue, Curanipe y Llico). Asimismo, sólo un tercio de los PRES de la Región del Maule y Bío Bío considera la relocalización de equipamiento clave y vivienda destruida por el maremoto desde zonas de riesgo hacia zonas seguras. En este contexto, es importante señalar, que en muchos casos la población no está dispuesta a ser relocalizada, resultando en litigios y dificultades con los títulos de dominio. Otro problema es que generalmente la relocalización es propuesta hacia zonas periféricas distantes, muy alejadas de los centros y las actividades económicas como son la pesca, el comercio y turismo.

Según la ONEMI (2001) una adecuada gestión frente a tsunamis debe insertarse en una planificación integral de protección civil comunal a cargo del Comité Local de Protección Civil, presidido por el Alcalde, coordinado por el Director de Protección Civil o Emergencia, e integrado por los organismos de la comuna y los representantes de la ciudadanía. En cuanto a la representación de la ciudadanía, se observa que todos los PRES declaran que la participación ciudadana constituye uno de los elementos más relevantes del proceso de reconstrucción. Así, los PRES fueron difundidos a través de diarios, internet, folletos informativos, posters y charlas informativas, destacándose el caso de Pelluhue con sus "caravanas informativas" y "mesas 
territoriales". También hubo instancias de participación consultiva como encuestas en terreno, talleres o foros participativos, así como asambleas.

Ahora, si bien los PRES contemplaron en su elaboración una participación ciudadana de modalidad informativa y consultiva, el proceso careció de una modalidad gestionaría, es decir, una efectiva participación de los líderes comunitarios y la comunidad en la toma de decisiones de políticas públicas. La ciudadanía fue informada y consultada, pero no se observa un proceso de negociación y toma de decisión en conjunto entre funcionarios públicos y la ciudadanía en la elaboración de los PRES. Asimismo, cabe destacar, que se han registrado casos de resistencia ciudadana frente a algunas medidas de mitigación de impacto de tsunami, como en el caso del Parque de Mitigación de Constitución. Con el objetivo de generar un parque de mitigación, se han expropiado 115 viviendas para ser relocalizadas desde la zona de riesgo de tsunami hacia zonas seguras, lo que alrededor de un 30\% de los propietarios está apelando en Corte. El costo del parque de mitigación incluyendo las expropiaciones y el diseño es de alrededor de 20.000 millones de pesos, financiado principalmente mediante fondos públicos. Cabe señalar, que el parque cubre sólo una pequeña parte del área de inundación por tsunami de Constitución, y que tiene un ancho insuficiente para mitigar el impacto de un maremoto de gran magnitud.

\section{Discusión y Conclusiones}

Esta investigación muestra que la elaboración de medidas de mitigación de impacto de tsunami en los recientes planes locales de reconstrucción se está realizando en un marco regulatorio nacional débil, dado que la única normativa relevante desarrollada en Chile a escala nacional tras la catástrofe del 27F es la modificación de la OGUC en el año 2011, que incluye por primera vez las zonas de inundación por tsunami como áreas de riesgo (D.S. N9, modificación OGUC). Sin embargo, la OGUC no establece parámetros para la ocupación y el manejo de zonas de riesgo por tsunami: no establece normas o estándares para guiar a los municipios en la planificación de zonas de riesgo por tsunami, ni restringe o prohíbe usos en éstas. Como consecuencia, y debido a la falta de regulación a escala nacional, las medidas de mitigación de impacto de tsunami difieren altamente entre los distintos PRES.

Así, a escala local se proponen, a través de los distintos PRES, medidas de mitigación de efectos de tsunami de carácter no vinculante, enfocadas principalmente en soluciones ingenieriles "anti-tsunámicas". Otros instrumentos de planificación como son la zonificación de riesgo - restringiendo, por ejemplo infraestructura clave en zonas de inundación por tsunami (colegios, hospitales, etc.) - o la relocalización de vivienda se han propuesto solamente en unos pocos PRES, sin instrumentos adecuados para su implementación y sin una participación ciudadana activa.

Cabe destacar, que las zonas de riesgo deben regularse a través de instrumentos vinculantes. Esto resulta fundamental, dado que en la definición de los usos de suelo costero participan numerosos actores con distintos intereses. Así, la única forma de asegurar el cumplimiento de las regulaciones establecidas para resguardar las áreas de riesgo es que las normativas sean de carácter obligatorio y vinculante. Al definirse para el manejo de zonas de riesgo recomendaciones de carácter no vinculante, éstas se convierten en meras "buenas intenciones", que no son implementadas y respetadas por los distintos actores, no lográndose así el objetivo de mejorar la resiliencia comunitaria costera. En conclusión, se requiere en Chile - tanto a escala nacional como local- de un marco regulatorio claro y vinculante que establezca objetivos generales así como usos permisibles y prohibidos para zonas de inundación por tsunami, para así poder reducir el riesgo y la vulnerabilidad de nuestros asentamientos costeros. 
Se observa en Chile hace décadas una conciencia sísmica, tanto a nivel de políticas públicas como de memoria colectiva, lo cual ha aumentado nuestra "resiliencia sísmica". Sin embargo, hasta hoy no contamos con políticas públicas adecuadas para reducir nuestra vulnerabilidad frente a la amenaza de tsunami. Este artículo revela que la planificación territorial en Chile no ha considerado suficientemente la amenaza de tsunami, pese a que Chile es un país muy expuesto a maremotos. Pareciera que en el caso de los tsunamis resultara particularmente problemática la elaboración e implementación de políticas públicas. Esto obedece por un lado a que la zona costera es un área de múltiples usos y fuertes conflictos ambientales y territoriales, donde es difícil consensuar normativas y regulaciones y donde además existe una fuerte presión por un desarrollo inmobiliario y turístico cerca de la línea de playa en las zonas de mayor riesgo (Eisner, 2005: pp.155). Otra razón por la cual resulta dificultosa la implementación de políticas públicas, es que los tsunamis destructivos ocurren en un mismo lugar cada 50 a 100 años, recurrencia menor que la de otros desastres naturales como son, por ejemplo, terremotos e inundaciones fluviales. Así, el mayor tiempo de retorno de tsunamis deriva en una menor conciencia política y colectiva acerca de la urgente necesidad de generar políticas públicas que reduzcan nuestra vulnerabilidad frente a los tsunami, que si bien ocurren con menor frecuencia que otros desastres naturales, son extremadamente destructivos y constituyen una amenaza para nuestras zonas costeras. La experiencia del 27F debe convertirse en aprendizaje social y políticas públicas, dado que sino sólo se transforma en "relatos efímeros, que no sólo son olvidados con prontitud, sino que además contribuyen a la adopción de decisiones improvisadas que terminan aumentando la vulnerabilidad ante eventos futuros" (Romero, 2012).

\section{Referencias}

Andrade, B., Arenas, F., \& Guijón, R. (2008). Revisión crítica del marco institucional y legal chileno de ordenamiento territorial: el caso de la zona costera. Revista de Geografía Norte Grande, 41, 2008, 23-48. https://doi.org/10.4067/s0718-34022008000300002

EERI Special Earthquake Report, Learning from Earthquakes, EERI. (2010). The Mw 8.8 Chile $\begin{array}{llllll}\text { Earthquake } & \text { of } & \text { February } & 2010 . & \text { Recuperado }\end{array}$ https://www.eeri.org/site/images/eeri_newsletter/2010_pdf/Chile10_insert.pdf

Eisner, R. (2005). Planning for tsunami: Reducing future losses through mitigation. Natural Hazards, 35(1), 155-162. https://doi.org/10.1007/s11069-004-2417-x

Federal Emergency Management Agency, FEMA. (2008). Guidelines for design of structures for vertical evacuation for tsunamis. Recuperado de https://www.fema.gov/medialibrary/assets/documents/14708

Forbes, K. \& Broadhead, J. (2007). The role of coastal forests in the mitigation of tsunami impacts. Recuperado de http://www.fao.org/forestry/14561-09bf06569b748c827dddf4003076c480c.pdf

Giménez, P., Pantoja, J., y Troncoso, C. (2010). Hacia un nuevo borde costero en Chile. Documento de Trabajo 58, Santiago.

I báñez, J. (1985). Historia y enseñanza de los terremotos. Revista CA, 42, 26-29.

Ministerio de Vivienda y Urbanismo, Minvu. (2010). Minuta Programa de reconstrucción en vivienda. Recuperado de http://ciperchile.cl/wp-content/uploads/minuta-reconstrucion.pdf 
Ministerio de Vivienda y Urbanismo, Minvu. (2013). NTM 007 Diseño estructural para edificaciones en zonas inundables por tsunami o seiche. http://proveedorestecnicos.minvu.cl/wpcontent/uploads/Norma-Tecnica-Minvu_007.pdf

Ministerio de Vivienda y Urbanismo, Minvu. (2011). Ley general de urbanismo y construcciones. Santiago de Chile: Autor.

Ministerio de Vivienda y Urbanismo, Minvu. (2011). Ordenanza general de urbanismo y construcciones. Santiago de Chile: Autor.

Ministerio de Vivienda y Urbanismo (2012). Actualización de instrumentos de planificación territorial. Recuperado de http://www.minvu.cl/opensite_20100901145818.aspx

Organisation for Economic Co-operation and Development, OECD. (2013). Urban policy reviews. Paris: OECD Publishing.

Oficina Nacional de Emergencias, ONEMI. (2001). Metodología básica para la elaboración de un plan comunal de respuesta ante tsunami. Santiago de Chile: Autor.

Romero, H. (2012). Los desastres naturales no naturales y la necesidad de conocer y comprender la vulnerabilidad social como uno de sus componentes principales. Recuperado de http://www.uchile.cl/noticias/80843/h-romero-desastres-naturales-no-naturales-y-lavulnerabilidad-social

Sarrazín, M. (1985). Regulaciones Sismorresistentes en Chile. Revista AUCA, 49, 25-28.

Servicio Hidrográfico y Oceanográfico de la Armada, SHOA. (2012). CITSU Cartas de inundación por tsunami. Recuperado de http://www.snamchile.cl/citsu/citsu_html

Servicio Hidrográfico y Oceanográfico de la Armada, SHOA. (s.f.). Tsunamis registrados en la costa de Chile. Recuperado de http://www.shoa.cl/servicios/tsunami/data/tsunamis historico.pdf

Tanaka, N., Sasaki, Y., Mowjood, M. I. M., Jinadasa, K. B. S. N., \& Homchuen, S. (2007). Coastal vegetation structures and their functions in tsunami protection. Landscape and Ecological Engineering, 3(1), 2007, 33-45. https://doi.org/10.1007/s11355-006-0013-9 